\title{
Gingival Thickness Assessment at Mandibular Incisors of Orthodontic Patients with Ultrasound and Cone-beam CT. A Cross-sectional Study
}

\author{
Dimitrios Kloukosa / Lydia Kakalib / George Koukosc / Anton Sculeand / Andreas Stavropoulos ${ }^{\mathrm{e}}$ / \\ Christos Katsaros ${ }^{f}$
}

Purpose: To use and evaluate two methods for measuring gingival thickness (GT) at mandibular incisors of orthodontic patients and compare their performance in assessing periodontal anatomy through soft tissue thickness.

\begin{abstract}
Materials and Methods: The sample consisted of 40 consecutive adult orthodontic patients. GT was measured just before bracket placement at both central mandibular incisors, mid-facially on the buccal aspect, $2 \mathrm{~mm}$ apically to the free gingival margin with two methods: clinically with an ultrasound device (USD) and radiographically with conebeam computed tomography (CBCT).

Results: CBCT measurements were consistently higher than USD measurements, with the difference ranging from $0.13 \mathrm{~mm}$ to $0.21 \mathrm{~mm}$. No statistically significant difference was noted between the repeated CBCT measurements at the right central incisor (bias $=0.05 \mathrm{~mm} ; 95 \% \mathrm{Cl}=-0.01,0.11 ; \mathrm{p}=0.104$ ). Although the respective results for the left incisor statistically indicated that the measurements were not exactly replicated, the magnitude of the point estimate was small and not clinically significant (bias $=0.06 \mathrm{~mm} ; 95 \% \mathrm{Cl}=0.01,0.11 ; p=0.014$ ). Small differences between CBCT measurements made by the 2 examiners at the left central incisor (bias $=0.06 \mathrm{~mm}$; $95 \%$ $\mathrm{Cl}=0.01,0.11 ; \mathrm{p}=0.014$ ) were detected. However, this difference was minor and also not clinically significant. The respective analysis on the right incisor showed no statistically significant difference (bias $=0.05 \mathrm{~mm}$; $95 \%$ $\mathrm{Cl}=-0.01,0.11 ; \mathrm{p}=0.246)$.
\end{abstract}

Conclusions: Based on reproducibility, CBCT imaging for gingival thickness assessment proved to be as reliable as ultrasound determination. However, CBCT consistently yielded higher values, albeit at a marginal level, than did the ultrasound device.

Key words: cone-beam CT, gingival phenotype, periodontal tissue, ultrasound

$\mathrm{T}$ he assessment of gingival thickness (GT) is often an important element that should be taken into consideration during treatment planning and subsequent decision making before various dental treatments. Quantitative and qualitative analysis of several periodontal parameters plays an important role, not only in planning periodontal proce-

\footnotetext{
a Lecturer, Department of Orthodontics and Dentofacial Orthopedics, School of Dental Medicine, University of Bern, Bern, Switzerland; Department of Orthodontics and Dentofacial Orthopedics, 251 Hellenic Air Force \& VA General Hospital, Athens, Greece. Performed all clinical and radiographic measurements, wrote the manuscript and prepared all figures, reviewed the manuscript.
}

${ }^{b}$ Researcher, Department of Orthodontics and Dentofacial Orthopedics, 251 Hellenic Air Force \& VA General Hospital, Athens, Greece. Performed all clinical and radiographic measurements, wrote the manuscript and prepared all figures, reviewed the manuscript

c Researcher, Department of Periodontology, 251 Hellenic Air Force \& VA Gen eral Hospital, Athens, Greece. Performed all clinical and radiographic measure ments, assisted with the interpretation of statistics, reviewed the manuscript. a Professor and Chairman, Department of Periodontology, School of Dental Medicine, University of Bern, Bern, Switzerland. Oversaw the project and assisted with the writing of the manuscript, reviewed the manuscript.

e Professor, Department of Periodontology, Faculty of Odontology, Malmö University, Malmö, Sweden; Professor, Division of Regenerative Dental Medicine and Periodontology, CUMD, University of Geneva, Geneva, Switzerland. Wrote the manuscript and prepared all figures, reviewed the manuscript.

$f$ Professor and Chairman, Department of Orthodontics and Dentofacial Orthopedics, School of Dental Medicine, University of Bern, Bern, Switzerland. Initiated and oversaw the project, assisted with the writing of the manuscript, reviewed the manuscript.

Correspondence: Dimitrios Kloukos, Department of Orthodontics and Dentofacial Orthopedics, University of Bern, Freiburgstrasse 7, CH-3010 Bern, Switzerland.Tel: +41-31-632-2592; e-mail: dimitrios.kloukos@zmk.unibe.ch 
dures, 10,16,19 but also in conventional prosthodontics, 24 implant therapy, 21,25,27 and orthodontics, when a change of tooth inclination is anticipated. 7,36

Several methods have been recommended for measuring GT. Visual appraisal of gingival phenotype may be considered as relatively uncomplicated and time-saving. However, it might not always be considered an objective method: it has been demonstrated that, irrespective of the clinician's skill, gingival phenotype is accurately identified in only about $50 \%$ of the cases. ${ }^{14}$ Another straightforward and commonly used method is transgingival probing with a periodontal probe. ${ }^{23}$ Potential limitations of this technique include the angulation of probe insertion, and the invasive nature of the procedure. Local anaesthesia is often required, which, in turn, has a two-fold limitation: patient discomfort and a transient local volume increase from injecting the local anaesthetic solution. 33 Transgingival probing with an endodontic file has been proposed to overcome patient discomfort; nevertheless, problems with this method's accuracy have been reported.22

An ultrasound device (USD) was proposed to resolve these limitations. ${ }^{13}$ The reproducibility of this method has been reported to be high. ${ }^{27}$ Another routinely applied procedure to classify gingival phenotype as thin or thick involves placement of a periodontal probe in the gingival sulcus; then, its transparency through the soft tissue is appraised. 20 This method has also been reported to be highly reproducible, with $85 \%$ agreement between duplicate measurements. 12

Finally, the use of cone-beam computed tomography (CBCT) has also shown a high diagnostic accuracy in assessing GT, demonstrating minimal discrepancy to clinical and radiographic measurements. 4,16,17 A few studies have suggested CBCT as a standard method for determining gingival and bone thickness. 1,8,16,29 Recently, technological developments have resulted in CBCT devices with lower radiation emissions, ${ }^{9}$ which renders it applicable in almost all dental procedures, although each patient should be evaluated individually based on their unique treatment needs and set of circumstances.

Nevertheless, it is still unclear which is the most suitable method for assessing GT. The lack of a gold standard technique to assess GT in human studies does not permit any clear recommendations for the clinician.

Since limited evidence-based data exist to validate the accuracy of CBCT in evaluating the thickness of soft tissues in the oral cavity, the aim of the present study was to compare CBCT with USD and determine the comparability and applicability of these methods as diagnostic tools for assessing GT on a subset of patients from a larger prospective study on orthodontic treatment, GT and the development of gingival recessions.

\section{MATERIALS AND METHODS}

\section{Ethics Approval and Consent to Participate}

All procedures were in accordance with the ethical standards of the institutional and national research committee and with the 1964 Helsinki declaration and its later amend- ments. The study protocol was approved by the 251 Greek Air Force Hospital's Education, Ethics and Research Committee (approval number: 076/7592/06.05.2015). All patients, or their legal guardian, provided written consent to participate prior to any measurements or performance of CBCT.

\section{Sample Selection}

This cross-sectional study clinically evaluated GT in 40 white (Caucasian; $\geq 16$ years old) orthodontic patients just before the commencement of orthodontic treatment, who consecutively visited the Department of Orthodontics and Dentofacial Orthopedics, 251 Hellenic Airforce Hospital, Athens, Greece.

CBCTs were not performed primarily for evaluating GT; CBCTs were principally carried out in order to assess bone condition and turnover in the mandibular anterior region, as part of an ongoing prospective controlled study assessing the occurrence of gingival recessions in orthodontically treated patients.

Exclusion criteria were: presence of crown restorations or fillings involving the cervical part of the anterior mandibular teeth, pregnant or lactating females, presence of obvious clinical signs of gingival conditions/diseases resulting in swelling of the gingiva (e.g. gingivitis), or presence of increased probing depths (e.g. $>3 \mathrm{~mm}$ ) at the mandibular central incisors, presence of labial gingival recessions at the mandibular central incisors, intake of medication with any known effect on the gingiva, (e.g. calcium antagonists, etc.), presence of congenital anomalies or dental structural disorders.

\section{Sample Size Calculation}

Although this cohort of patients derived from another ongoing, prospective study assessing gingival recessions in orthodontically treated patients, a current sample size calculation was performed using the formula of comparing two means, and included 90\% power and statistical significance of 0.05 . The standard deviation applied was $0.18 \mathrm{~mm}$ according to previous research 22 and the anticipated mean difference was $0.20 \mathrm{~mm}$.

\section{Clinical and Measuring Parameters}

All clinical procedures, as well as CBCT imaging, were performed before bracket placement. Measurements were carried out at both central mandibular incisors, mid-facially on the buccal aspect of each tooth, and $2 \mathrm{~mm}$ apical to the free gingival margin, with the following two methods.

\section{Ultrasound}

A periodontist (GK) assessed the GT of each patient with the ultrasound device (USD; Krupp SDM, Austenal Medizintechnik; Cologne, Germany). Measuring GT with USD is based on the ultrasonic pulse-echo principle: ultrasonic pulses are transmitted through the sound-permeable tissue $(1518 \mathrm{~m} / \mathrm{s})$ and are reflected at the surface of the hard tissue. By timing the received echo, GT is determined and digitally displayed. Measurements may range between 0.5 and $8.0 \mathrm{~mm}$ with a resolution of $0.1 \mathrm{~mm}$. Ultrasonic frequency is 


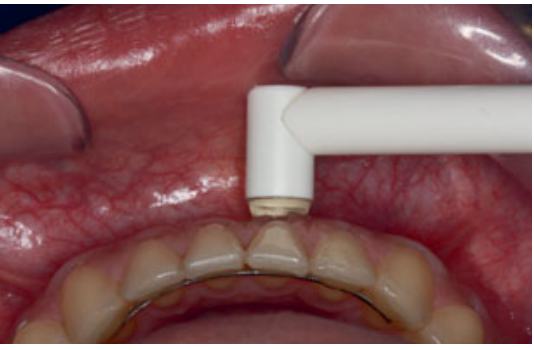

Fig 1 Measurement procedure with the ultrasound device.
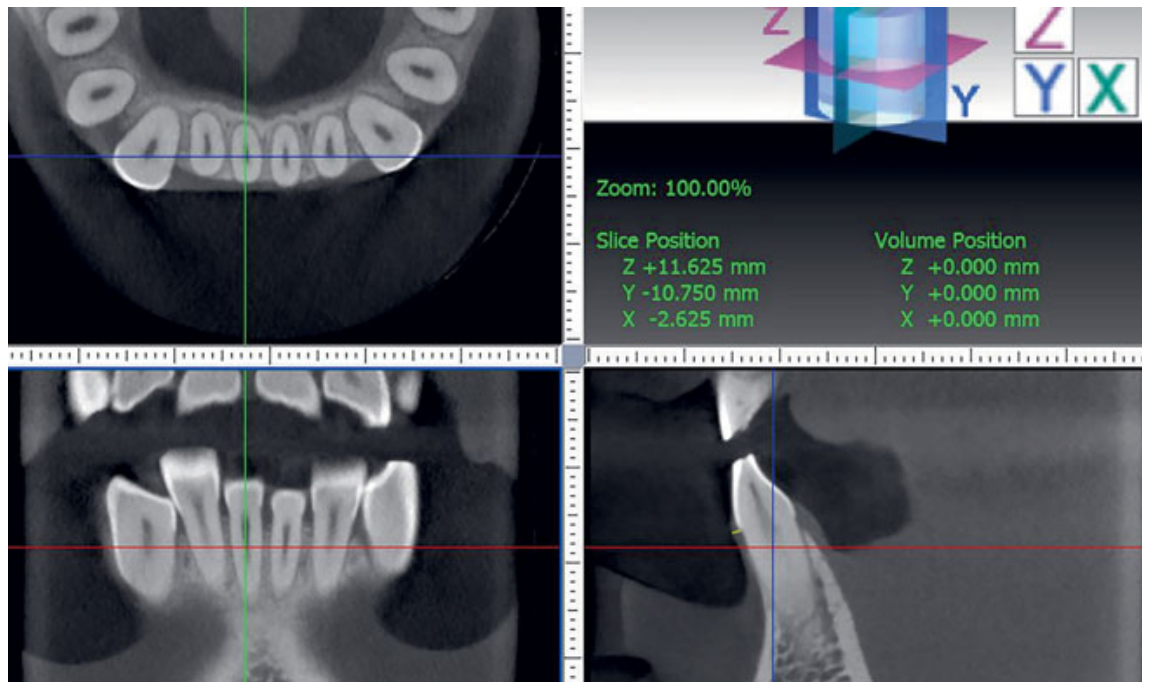

Fig 2 Measurement procedure in the CBCT images.
$5 \mathrm{MHz}$ and the diameter of the transducer probe is $3 \mathrm{~mm}$ with a weight of $19 \mathrm{~g}$. Measurements were performed by perpendicularly placing the transducer probe on the gingival surface without pressure, ensuring that the center of the transducer was $2 \mathrm{~mm}$ apical to the free gingival margin (Fig 1).

\section{CBCT imaging}

All patients underwent CBCT examination in a private clinic (Orofacial Radiodiagnosis; Athens, Greece). CBCT images were acquired using the Morita Accuitomo 80 3D Imaging System (J. Morita; Darmstadt, Germany) at $90 \mathrm{kV}$ and $7 \mathrm{~mA}$ for $17.5 \mathrm{~s}$ and a single 360-degree image rotation. The CBCT scans were obtained with $6 \times 6 \mathrm{~cm}$ field of view and $80 \mu$ voxel size. Images were processed by I-Dixel-3DX software, v 2.0 (J. Morita; Darmstadt, Germany). During the examination, a cotton roll was used to retract the lip and enable the imaging of labial soft tissues.

\section{GT Measurement using CBCT Imaging}

The GT in all CBCT images was measured independently by two authors (DK, LK) and recorded in data extraction forms without patient identification information. The first examiner (DK) conducted the measurements twice with an intermediate interval of one month in order to evaluate the intra-examiner repeatability.

The method for measuring GT in the software was standardised after calibration between the two assessors using ten randomly selected CBCTs. This was done to ensure reproducibility of the measurement location $(2 \mathrm{~mm}$ apical to the free gingival margin), as was the case for the clinical measurements with the ultrasound transducer probe. Measurements in the CBCT images were then performed perpendicularly to the tooth axis (Fig 2).

\section{Statistical Analysis}

Descriptive statistics were performed for age, CBCT and USD measurements of gingival thickness. The repeated CBCT measurements by the first examiner were tested for systematic differences (bias) using paired t-tests. Repeatability was quantified via the $95 \%$ repeatability coefficient.5,6 The presence of a magnitude-related trend for the differences as well as for their dispersion was assessed graphically. Additionally, the presence of a trend for the differences was assessed statistically using Spearman's rank correlation coefficient. The normality assumption was examined both graphically and via the Shapiro-Wilk test. The agreement between the two examiners on GT measurements from CBCT data was assessed both statistically and graphically. Paired t-tests were applied to test for systematic differences between the two examiners, while the reproducibility was quantified via the $95 \%$ reproducibility coefficient and in accordance with the repeatability coefficient. Again, normality assumptions and magnitude-related trends were evaluated as above. Method agreement was evaluated between CBCT and USD measurements using two separate Bland-Altman analyses. Finally, the 95\% Limits of Agreement (95\% LOA) and the corresponding 95\% Cls were calculated. Normality assumptions were evaluated graphically and by means of the Shapiro-Wilk test. Statistical significance was set to $\alpha=5 \%$. All statistical analyses and graphical plots were conducted or constructed, respectively, using Stata 13.0/SE software (StataCorp; College Station, TX, USA). 
Table 1 Descriptive statistics of all gingival thickness measurements (in $\mathrm{mm}$ )

\begin{tabular}{|c|c|c|c|}
\hline & Mean (SD) & Min & Max \\
\hline Age & $24.48(6.68)$ & 18.00 & 45.00 \\
\hline \multicolumn{4}{|c|}{ Mandibular left central incisor } \\
\hline СВCT: Examiner \#1 & $0.93(0.24)$ & 0.55 & 1.51 \\
\hline СВCT: Examiner \#2 & $1.01(0.24)$ & 0.61 & 1.59 \\
\hline USD & $0.80(0.26)$ & 0.50 & 1.50 \\
\hline \multicolumn{4}{|c|}{ Mandibular right central incisor } \\
\hline СВСТ: Examiner \#1 & $0.95(0.27)$ & 0.50 & 1.50 \\
\hline СBCT: Examiner \#2 & $0.99(0.25)$ & 0.42 & 1.43 \\
\hline USD & $0.80(0.22)$ & 0.50 & 1.50 \\
\hline
\end{tabular}

Table 2 Results of the paired t-tests between the repeated CBCT measurements by examiner \#1

\begin{tabular}{|llll|}
\hline Tooth & Bias (SE) & $95 \% \mathrm{Cl}$ & $\mathrm{p}$-value \\
\hline Mandibular left central incisor & $0.06(0.02)$ & $(0.01,0.11)$ & 0.014 \\
\hline Mandibular right central incisor & $0.05(0.03)$ & $(-0.01,0.11)$ & 0.104 \\
\hline Bias = 2nd 1st measurements (in mm) & & & \\
\hline SE: standard error. & & & \\
\hline
\end{tabular}

\section{RESULTS}

Forty subjects (17 females and 23 males) participated in this study. The descriptive statistics for age, CBCT and USD measurements are reported in Table 1.

\section{Repeatability Assessment}

The results of the paired t-tests for bias between the 1st and the 2nd СВCT measurements made by the first examiner (DK) are reported in Table 2. Repeatability of USD measurements were performed in a previous cross-sectional study with the same methodology and objective. 22 The normality assumption was not violated for any of the differences between the repeated measurements of USD at two time points (mean difference $0.00 ; 95 \% \mathrm{Cl}-0.05,0.05 ; \mathrm{p}=1.00$ ).

Statistical analysis indicated that the repeated CBCT measurements were not identical for the mandibular left central incisor (bias $=0.06 \mathrm{~mm} ; 95 \% \mathrm{Cl}=0.01,0.11 ; \mathrm{p}=0.014$ ), whereas the repeated CBCT measurements for the mandibular right central incisor could be considered identical (bias $=0.05 \mathrm{~mm} ; 95 \% \mathrm{Cl}=-0.01,0.11 ; \mathrm{p}=0.104$ ). Nevertheless, a difference of $0.06 \mathrm{~mm}$ in repeated measurements can be generally regarded as clinically irrelevant. The corresponding Bland-Altman plots are displayed in Fig $3 a$ and $3 \mathrm{~b}$. Neither a magnitude- nor a dispersion-related trend could be identified graphically. An absence of a magnitude trend was also implied by the Spearman's rank correlation coefficient (mandibular left central incisor: Spearman's rho $=0.10$, $p$-value $=0.554$; mandibular right central incisor: Spearman's rho $=-0.14, p=0.377$ ). The Shapiro-Wilk test results showed that the normality hypothesis was valid for both left and right mandibular incisors $(p=0.595$ and 0.614 , respectively).

\section{Reproducibility Assessment}

The results of the paired t-tests for bias between the two examiners (DK, LK) are reported in Table 3. Again, statistical analysis indicated that the repeated measurements were not identical for the mandibular left central incisor (bias $=0.06 \mathrm{~mm} ; 95 \% \mathrm{Cl}=0.01,0.11 ; \mathrm{p}=0.014$ ), whereas the repeated measurements for the mandibular right central incisor could be considered identical (bias $=0.05 \mathrm{~mm}$; 95\% $\mathrm{Cl}=-0.01,0.11 ; \mathrm{p}=0.246)$. Nevertheless, a difference of $0.06 \mathrm{~mm}$ in different operators' measurements can be generally regarded as clinically irrelevant. The corresponding Bland-Altman plots are displayed in Figs 3c and 3d. Neither a magnitude- nor a dispersion-related trend was identified (mandibular left central incisor: Spearman's rho $=-0.03$, $\mathrm{p}=0.836$; mandibular right central incisor: Spearman's rho $=-0.12, p=0.377)$. The normality hypothesis was valid 


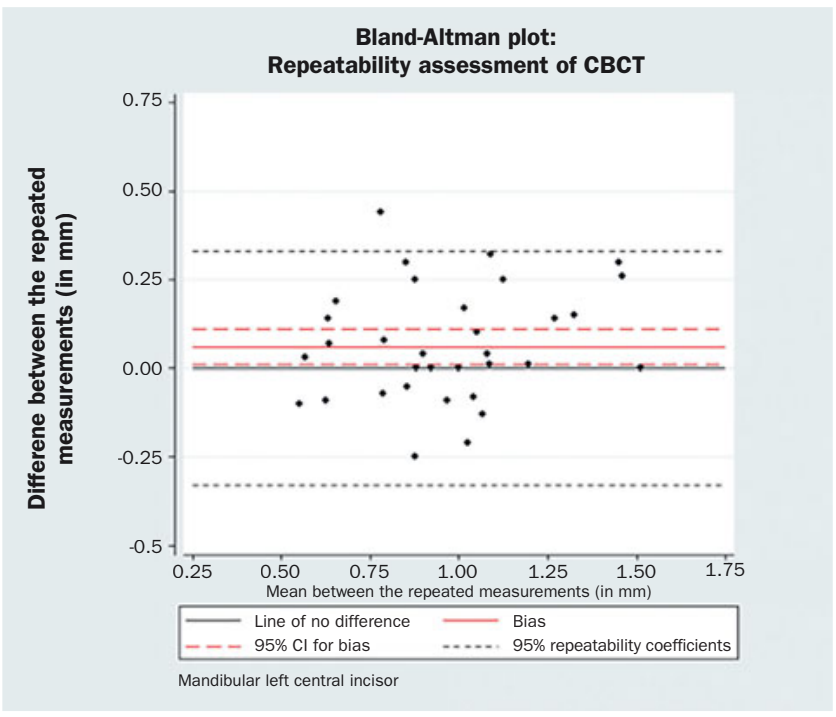

Fig 3a Bland-Altman plot: repeatability assessment for CBCT measurements, mandibular left central incisor.

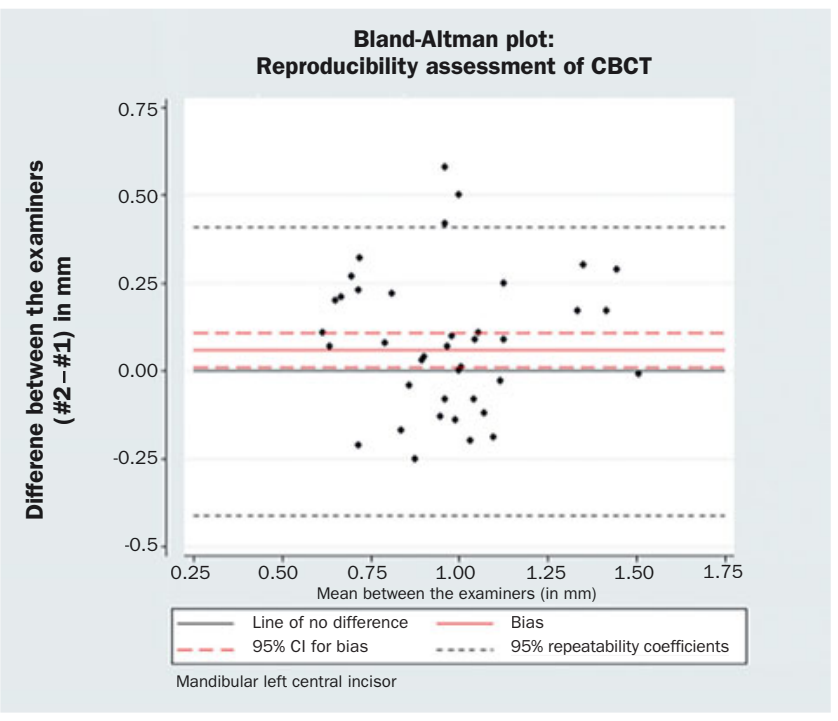

Fig 3c Bland-Altman plot: reproducibility assessment for CBCT measurements, mandibular left central incisor.

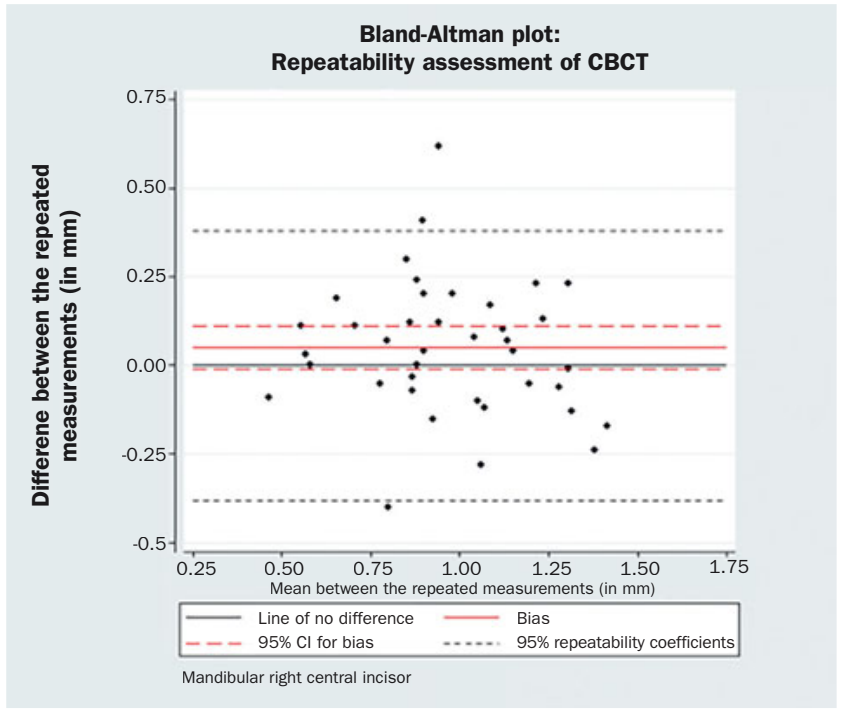

Fig 3b Bland-Altman plot: repeatability assessment for CBCT measurements, mandibular right central incisor.

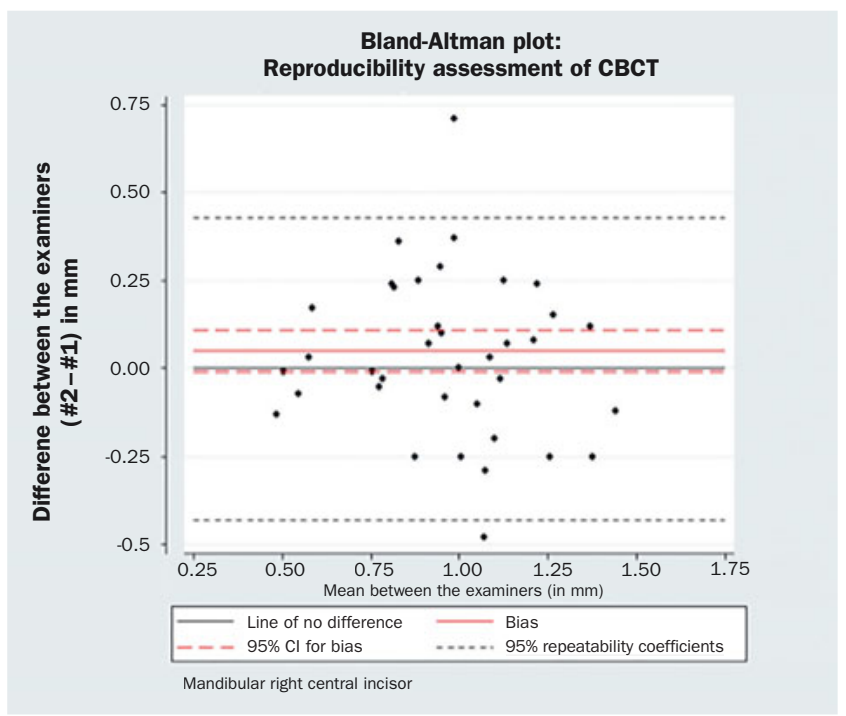

Fig 3d Bland-Altman plot: reproducibility assessment for CBCT measurements, mandibular right central incisor.

Table 3 Results of the paired t-tests between the CBCT measurements made by the two examiners

\begin{tabular}{|lccc|}
\hline Tooth & Bias (SE) & $95 \% \mathrm{Cl}$ & $\mathrm{p}$-value \\
\hline Mandibular left central incisor & $0.06(0.02)$ & $(0.01,0.11)$ & 0.014 \\
\hline Mandibular right central incisor & $0.05(0.03)$ & $(-0.01,0.11)$ & 0.246 \\
\hline Bias = examiner \#2 - examiner \#1 (in mm) & & & \\
\hline SE: standard error. & & & \\
\hline
\end{tabular}


Table 4 Results of the paired t-tests (bias, SE, 95\% Cl, p-value) between the CBCT and USD measurements, as well as the corresponding 95\% LOA and the respective 95\% $\mathrm{Cl}$ for the LOA

\begin{tabular}{|c|c|c|c|c|c|c|}
\hline & \multirow[t]{2}{*}{ Bias (SE) } & \multirow[t]{2}{*}{$\begin{array}{l}95 \% \mathrm{Cl} \\
\text { for bias }\end{array}$} & \multirow[t]{2}{*}{ p-value } & \multirow[t]{2}{*}{ 95\% LOA } & \multicolumn{2}{|c|}{$\begin{array}{c}95 \% \mathrm{Cl} \\
\text { for the LOA }\end{array}$} \\
\hline & & & & & Lower LOA & Upper LOA \\
\hline \multicolumn{7}{|l|}{ Mandibular left central incisor } \\
\hline CBCT measurements from examiner \#1 & $0.13(0.03)$ & $(0.07,0.19)$ & $<0.001$ & $(-0.26,0.51)$ & $(-0.35,-0.17)$ & $(0.42,0.60)$ \\
\hline CBCT measurements from examiner \#2 & $0.21(0.04)$ & $(0.14,0.28)$ & $<0.001$ & $(-0.22,0.64)$ & $(-0.32,-0.12)$ & $(0.54,0.75)$ \\
\hline \multicolumn{7}{|l|}{ Mandibular right central incisor } \\
\hline CBCT measurements from examiner \#1 & $0.14(0.03)$ & $(0.08,0.21)$ & $<0.001$ & $(-0.26,0.54)$ & $(-0.35,-0.16)$ & $(0.45,0.63)$ \\
\hline CBCT measurements from examiner \#2 & $0.18(0.04)$ & $(0.11,0.25)$ & $<0.001$ & $(-0.25,0.62)$ & $(-0.36,-0.15)$ & $(0.52,0.72)$ \\
\hline Bias = CBCT - USD (in mm). & & & & & & \\
\hline
\end{tabular}

for both left and right mandibular central incisors (ShapiroWilk test $p=542$ and 0.475 , respectively).

\section{Method Agreement (Comparability)}

The results of the paired t-tests between the two GT measuring techniques as well as the estimated corresponding 95\% LOA and the respective 95\% Cls are reported in Table 4. The respective Bland-Altman plots are displayed in Figs $4 \mathrm{a}$ to $4 \mathrm{~d}$. There was no evidence of a magnitude-related trend for either the differences or their dispersion after graphical evaluation.

Finally, none of the normality assumptions could be rejected after either graphical evaluation or using Shapiro-Wilk tests (mandibular left central incisor, CBCT measurements from examiner \#1: $p=0.163$; CBCT measurements from examiner \#2: $p=0.561$; mandibular right central incisor, CBCT measurements from examiner \#1: $p=0.157$; CBCT measurements from examiner \#2: $p=0.097$ ).

\section{DISCUSSION}

The objective of the present study was to assess GT with non-invasive methods. Mandibular incisors were in focus, since a change in their inclination or root torque may introduce a risk factor for gingival recessions, making this an area of major concern in both functional and aesthetic respects.

Direct measurement with either a periodontal probe or an endodontic file is regarded as a fairly objective method for GT assessment. However, since it involves tissue penetration, its clinical application is associated with some limitations. ${ }^{37}$ These are often linked with measurement errors, probably originating from instruments' rounded tips and thickness. ${ }^{22}$

An USD showing high reproducibility13,22,27,28 was selected as the first non-invasive method for measuring GT.
The second selected method was CBCT imaging, which that has been shown to have high diagnostic applicability. 4,16

The present results show that the difference between USD and CBCT measurements of gingival thickness was not zero. CBCT measurements were constantly higher than those obtained with the USD, with the difference ranging from $0.13 \mathrm{~mm}$ to $0.21 \mathrm{~mm}$ (Table 4). This difference was independent of the magnitude of GT measurement. Although it is difficult to assign the difference reported to one methodology or the other, the difference could possibly be attributed to the ultrasound procedure, due to measuring imprecision such as misangulation of the ultrasound transducer or over-compression of the soft tissue.

There were no statistically significant differences between the repeated measurements made by the first examiner on the mandibular right central incisor $(p=0.104)$. Although the respective results for the mandibular left central incisor indicated that the measurements were not exactly true replicates from a statistical point of view, the magnitude of the point estimate of bias was small and possibly not clinically significant (bias $=0.06 \mathrm{~mm} ; \mathrm{p}=0.014$ ).

Moreover, there was evidence of a small systematic difference between the CBCT measurements made by the two examiners on the mandibular left central incisor (bias = $0.06 \mathrm{~mm}, 95 \% \mathrm{Cl}=0.01,0.11)$. However, this difference was minor, and again, clinically unimportant. On the other hand, the respective analysis on the mandibular right central incisor showed no statistically significant difference between the two examiners (bias $=0.05 \mathrm{~mm}, 95 \% \mathrm{Cl}=-0.01,0.11$ ).

Numerous dental procedures require accurate measurement of GT, since respect of the gingival phenotype is vital and appears to influence the outcomes of various treatment strategies. Gingival phenotype evaluation through simple visual appraisal is inaccurate, 11,14 mostly due to its subjective nature; to a great extent, it relies on clinical com- 


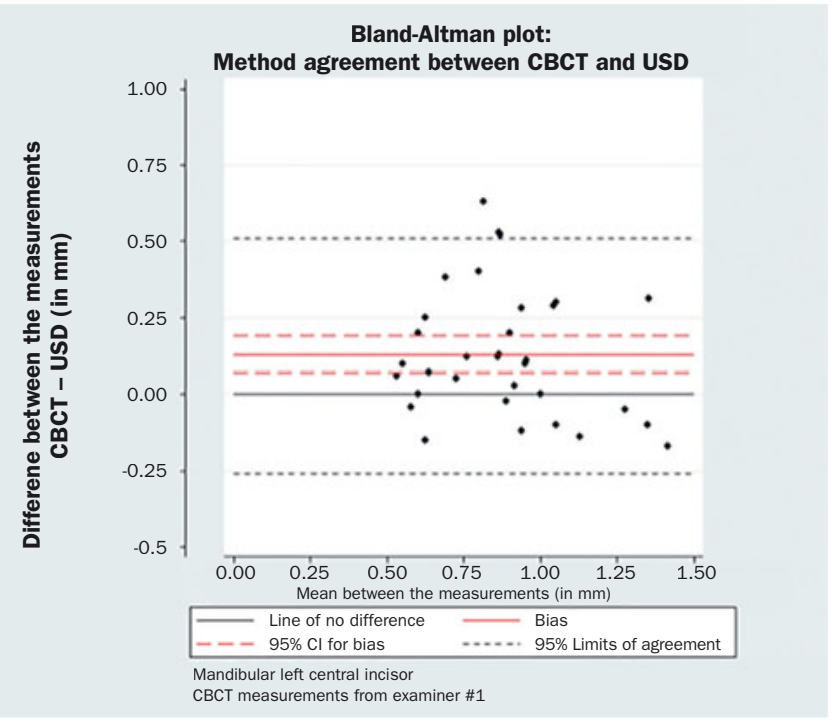

Fig 4a Bland-Altman plot: method agreement. Examiner \#1, mandibular left central incisor.

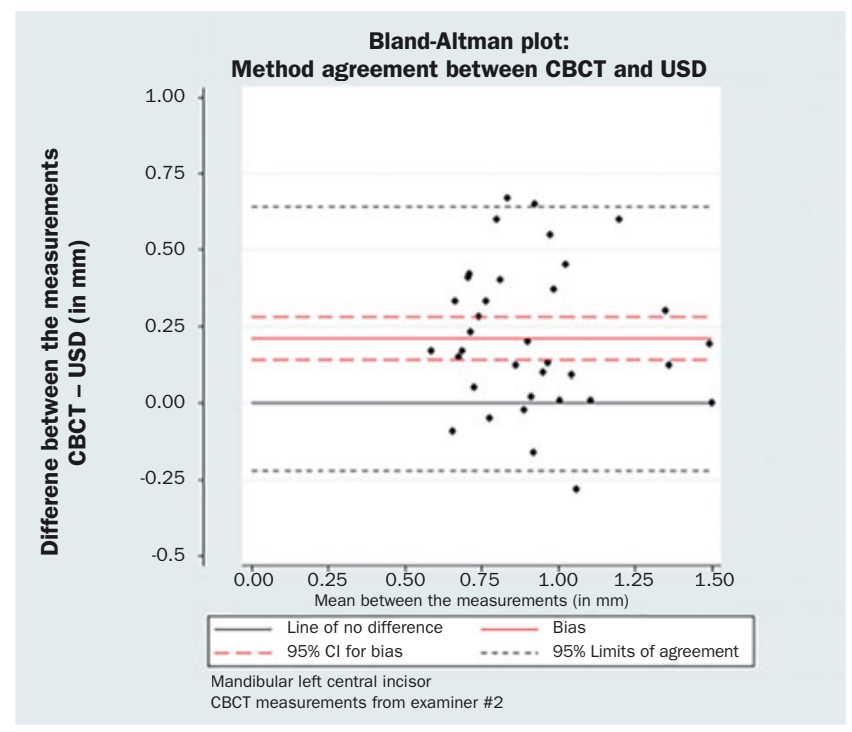

Fig 4c Bland-Altman plot: method agreement. Examiner \#2, mandibular left central incisor.

petence. Thick gingiva, i.e. $>0.81 \mathrm{~mm}$ thick, is shown to be relatively resistant to gingival recession following surgical or restorative therapies, 2,3,15,31 whereas thin-scalloped gingiva is considered at risk, because it has been associated with a compromised response following the same treatments. $2,2,15,25,30-32$ These findings point clearly to the need for a thorough diagnosis, through a straightforward and reproducible method, of these high-risk patients, prior to various interventions involving the gingiva. As far as accuracy is concerned, it is important to bear in mind that this term

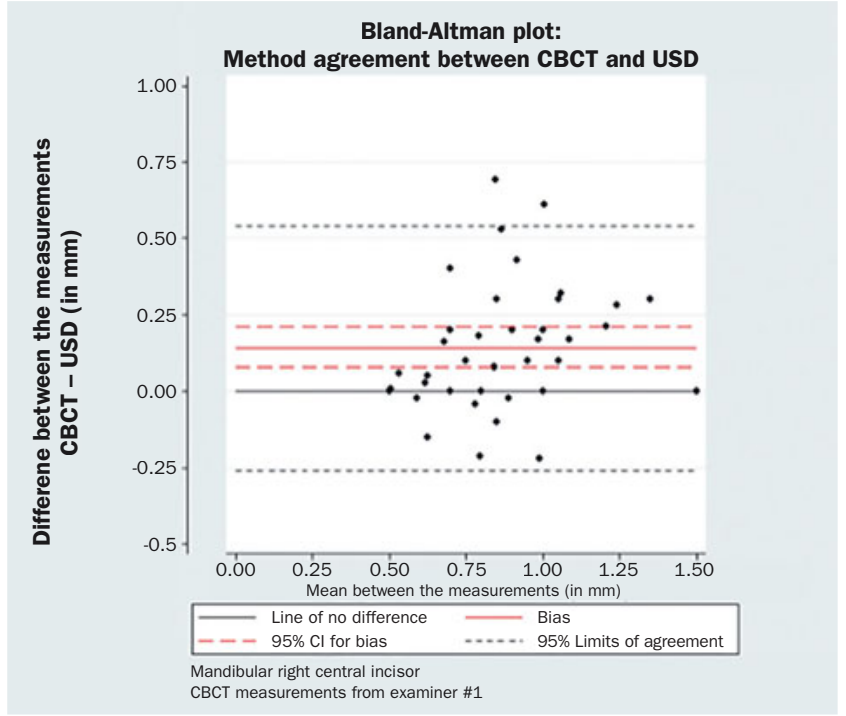

Fig 4b Bland-Altman plot: method agreement. Examiner \#1, mandibular right central incisor.

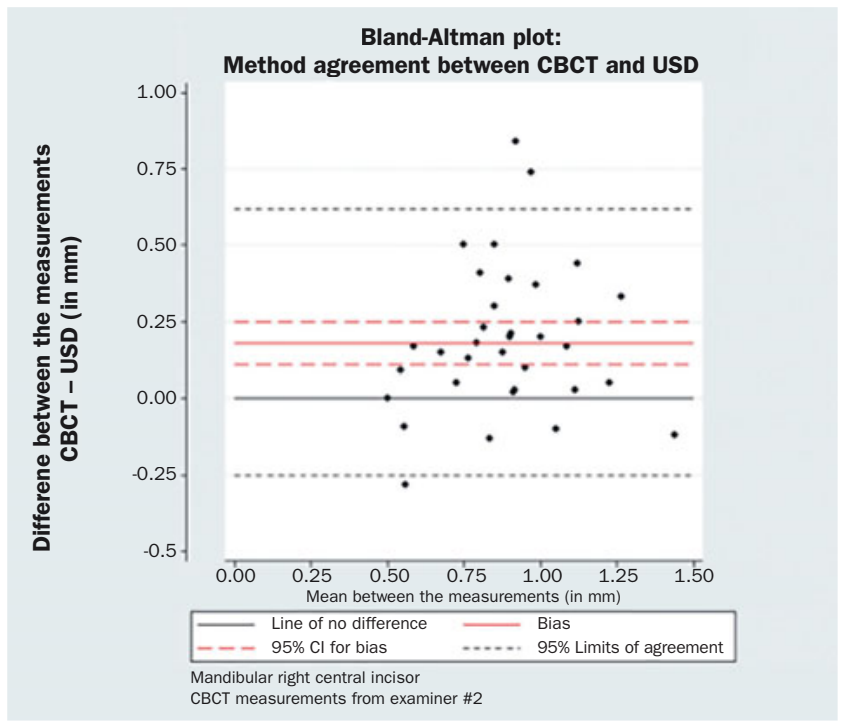

Fig 4d Bland-Altman plot: method agreement. Examiner \#2, mandibular right central incisor.

refers to the proximity of the measurements to the true value of gingival thickness. By definition, the true value cannot be measured by methods such as those in the present study. Only an estimation of the true value is possible. This is the reason why it is important to describe repeatability, reproducibility and the correlation of the methods tested.

Our study is not free of limitations, although efforts were made to minimise them. Firstly, the clinical measurements did not take into account potential differences in dental arch crowding or tooth inclination that may influence the 
clinical handling of the USD transducer probe, although it was expected that this would not lead to a large method error. Secondly, at present, conducting CBCT to assess GT might not be justified due to the amount of radiation, as CBCT involves higher doses than two-dimensional imaging. Moreover, CBCT images have a certain degree of inaccuracy, attributed primarily to image generation, processing, voxel size and various types of artefacts that might be present. In general, the smaller the voxel size, the higher the precision/ resolution of the information provided. Larger voxels may include different tissues, so that the subsequent grayscale value may not clearly indicate one specific tissue, such as bone. This issue is primarily evident at the limits between neighbouring tissue types of different radiodensity. However, at the same time, the smaller the voxel size, the higher the motion artefacts. Thus, based on the above considerations and also on the need to keep radiation exposure as low as possible, a specific CBCT image can only reach a certain degree of detail of the information it provides. $18,34,35$

Finally, it has to be pointed out that the lack of a gold standard procedure for measuring soft tissue thickness may downgrade the clinical significance. However, finding a gold standard measurement for humans is almost impossible, because all clinical or imaging methods present an inherent measurement error which is not always easy to assess during implementation. On the other hand, the relevance of the current study lies in the fact that it includes both imaging and clinical procedures and provides robust data for the comparison of the tested methods.

\section{CONCLUSIONS}

Based on reproducibility, CBCT imaging for gingival thickness assessment proved to be as reliable as ultrasound determination. However, CBCT imaging yielded consistently higher values, albeit at a marginal level, than did the ultrasound device.

\section{REFERENCES}

1. Amid R, Mirakhori M, Safi Y, Kadkhodazadeh M, Namdari M. Assessment of gingival biotype and facial hard/soft tissue dimensions in the maxillary anterior teeth region using cone beam computed tomography. Arch Oral Biol 2017;79:1-6.

2. Anderegg CR, Metzler DG, Nicoll BK. Gingiva thickness in guided tissue re generation and associated recession at facial furcation defects. J Periodontol 1995;66:397-402.

3. Baldi C, Pini-Prato G, Pagliaro U, Nieri M, Saletta D, Muzzi L, et al. Coronally advanced flap procedure for root coverage. Is flap thickness a relevant predictor to achieve root coverage? A 19-case series. J Periodontol 1999;77: 1077-1084.

4. Benavides E, Rios HF, Ganz SD, An CH, Resnik R, Tieszen Reardon G, et al. Use of cone beam computed tomography in implant dentistry: the International Congress of Oral Implantologists consensus report. Implant Dent 2012;21:78-86.

5. Bland JM, Altman DG. Measuring agreement in method comparison studies. Stat Methods Med Res 1999;8:135-160.

6. Bland JM, Altman DG. Applying the right statistics. Analyses of measurement studies. Ultrasound Obstet Gynecol 2003;22:85-93.

7. Boke F, Gazioglu C, Akkaya S, Akkaya M. Relationship between orthodontic treat ment and gingival health: A retrospective study. Eur J Dent 2014;8:373-380.
8. Borges GJ, Ruiz LF, de Alencar AH, Porto OC, Estrela C. Conebeam computed tomography as a diagnostic method for determination of gingival thickness and distance between gingival margin and bone crest. Sci World J 2015;142108.

9. Cesur E, Orhan K, Misirli M, Bilecenoglu B. Cone beam computed tomography evaluation of the relationship between atlantodental interval and skeletal facial morphology in adolescents. Braz J Otorhinolaryngol 2019; S1808-8694(19)30057-6.

10. Cook DR, Mealey BL, Verrett RG, Mills MP, Noujeim ME, Lasho DL, et al. Relationship between clinical periodontal biotype and labial plate thickness: an in vivo study. Int J Periodontics Restorative Dent 2011;31:345-354.

11. Cuny-Houchmand M, Renaudin S, Leroul M, Planche L, Guehennec LL, Soueidan A. Gingival biotype assessement: visual inspection relevance and maxillary versus mandibular comparison. Open Dent J 2013;7:1-6.

12. De Rouck T, Eghbali R, Collys K, De Bruyn H, Cosyn J. The gingival biotype revisited: transparency of the periodontal probe through the gingival margin as a method to discriminate thin from thick gingiva. J Clin Periodontol 2009;36:428-433.

13. Eger T, Müller HP, Heinecke A. Ultrasonic determination of gingival thickness. Subject variation and influence of tooth type and clinical features. J Clin Periodontol 1996;23:839-845.

14. Eghbali A, De Rouck T, De Bruyn H, Cosyn J. The gingival biotype assessed by experienced and inexperienced clinicians. J Clin Periodontol 2009;36: 958-963.

15. Evans CD, Chen ST. Esthetic outcomes of immediate implant placements. Clin Oral Impl Res 2008;19:73-80.

16. $\mathrm{Fu} \mathrm{JH}$, Yeh $\mathrm{CY}$, Chan HL, Tatarakis N, Leong DJ, Wang HL. Tissue biotype and its relation to the underlying bone morphology. J Periodontol 2010,81:569-574.

17. Gkogkos A, Kloukos D, Koukos G, Liapis G, Sculean A, Katsaros C. Clinical and radiographic gingival thickness assessment at mandibular incisors: an ex vivo study. Oral Health Prev Dent 2020;18:607-617.

18. Harris D, Horner K, Gröndahl K, Jacobs R, Helmrot E, Benic GI, et al. E.A.O. guidelines for the use of diagnostic imaging in implant dentistry 2011. A consensus workshop organized by the European Association for Osseointegration at the Medical University of Warsaw. Clin Oral Impl Res 2012;23: 1243-1253.

19. Hwang D, Wang HL. Flap thickness as a predictor of root coverage: a systematic review. J Periodontol 2006;77:1625-1634.

20. Kan JY, Rungcharassaeng K, Umezu K, Kois JC. Dimensions of peri-implant mucosa: an evaluation of maxillary anterior single implants in humans. J Periodontol 2003;74:557-562.

21. Kao RT, Fagan MC, Conte GJ. Thick vs. thin gingival biotypes: a key determinant in treatment planning for dental implants. J Calif Dent Assoc 2008;36: 193-198.

22. Kloukos D, Koukos G, Doulis I, Sculean A, Stavropoulos A, Katsaros C. Gingival thickness assessment at the mandibular incisors with four methods: A crosssectional study. J Periodontol 2018;89:1300-1309.

23. Kloukos D, Koukos G, Gkantidis N, Sculean A, Katsaros C, Stavropoulos A. Transgingival probing: a clinical gold standard for assessing gingival thickness. Quintessenz Int 2021, doi: 10.3290/j.qi.b937015

24. Kois JC. The restorative-periodontal interface: biological parameters. Periodontol 2000 1996;11:29-38.

25. Kois JC. Predictable single-tooth peri-implant esthetics: five diagnostic keys. J Calif Dent Assoc 2004;25:895-896.

26. Lee A, Fu JH, Wang HL. Soft tissue biotype affects implant success. Implant Dent 2011;20:e38-47.

27. Müller HP, Barrieshi-Nusair KM, Könönen E. Repeatability of ultrasonic determination of gingival thickness. Clin Oral Investig 2007;11:439-442.

28. Müller HP, Eger T. Gingival phenotypes in young male adults. J Clin Periodontol 1997;24:65-71.

29. Nikiforidou M, Tsalikis L, Angelopoulos C, Menexes G, Vouros I, Konstantinides A. Classification of periodontal biotypes with the use of CBCT. A cross-sectional study. Clin Oral Investig 2016;20:2061-2071.

30. Olsson $\mathrm{M}$, Lindhe J. Periodontal characteristics in individuals with varying form of the upper central incisors. J Clin Periodontol 1991;18:78-82.

31. Pontoriero R, Carnevale G. Surgical crown lengthening: a 12-month clinical wound healing study. J Periodontol 2001;72:841-848.

32. Romeo E, Lops D, Rossi A, Storelli S, Rozza R, Chiapasco M. Surgical and prosthetic management of interproximal region with single-implant restorations: 1-year prospective study. J Periodontol 2008;79:1048-1055.

33. Ronay V, Sahrmann P, Bindl A, Attin T, Schmidlin PR. Current status and perspectives of mucogingival soft tissue measurement methods. J Esthet Restor Dent 2011;23:146-156.

34. Spin-Neto R, Gotfredsen E, Wenzel A. Impact of voxel size variation on CBCTbased diagnostic outcome in dentistry: a systematic review. J Digit Imaging. 2013;26:813-820.

35. Spin-Neto R, Wenzel A. Patient movement and motion artefacts in cone beam computed tomography of the dentomaxillofacial region: a systematic literature review. Oral Surg Oral Med Oral Pathol Oral Radiol 2016;121:425-433.

36. Zawawi KH, Al-Zahrani MS. Gingival biotype in relation to incisors' inclination and position. Saudi Med J 2014;35:1378-1383.

37. Zweers J, Thomas RZ, Slot DE, Weisgold AS, Van der Weijden F. Characteristics of periodontal biotype, its dimensions, associations and prevalence: a systematic review. J Clin Periodontol 2014;41:958-971. 